\title{
Evaux Spring Water-based Cream
}

National Cancer Institute

\section{Source}

National Cancer Institute. Evaux Spring Water-based Cream. NCI Thesaurus. Code C120212.

A cream containing Evaux thermal spring water, with potential moisturizing and skin protecting activities. Upon application to the skin, Evaux spring water-based cream forms a protective barrier, which prevents water loss, provides moisture to the skin, protects the skin from damage, and soothes irritated skin. The Evaux thermal spring water contains the trace elements lithium and mang anese which may help heal the skin. 